\title{
Discrete Nonlinear Inequalities and Applications to Boundary Value Problems for Difference Equations
}

\author{
Baoju Sun \\ Zhejiang Water Conservancy \& Hydropower University, Hangzhou, 310018, China \\ sunbj@zjwchc.com
}

\begin{abstract}
In this paper, some new discrete nonlinear inequalities with two variables are established and explicit bounds on the unknown function are derived. These inequalities generalize former results and can be used as handy tools to study the qualitative as well as the quantitative properties of certain partial difference equations. Example of applying these inequalities to derive the properties of boundary Value problems for difference equations is also given.

Index Terms - Nonlinear Discrete Inequalities, Explicit Bound, Boundary Value Problems.
\end{abstract}

\section{I . Introduction}

Linear and nonlinear discrete inequalities in one and more than one independent variables which provide explicit bounds on unknown function play a fundamental role in the development of the theory of finite difference equations. During the past few years there have been a number of papers written on discrete versions of Gronwall-Bellman-type inequalities. For example, see [1-13]. Found in [6], the unknown function $u$ in the fundamental form of sumdifference inequality

$u(n) \leq a(n)+\sum_{s=0}^{n-1} b(s) u(s)$

can be estimated by

$u(n) \leq a(n) \prod_{s=0}^{n-1}(1+b(s)) \leq a(n) \exp \left(\sum_{s=0}^{n-1} b(s)\right)$

Where $u(n), a(n), b(n)$ are nonnegative functions, $a(n)$ is nondecreasing. Pang and Agarwal [2] considered the inequality

$$
u^{2}(n) \leq p^{2} u(0)+2 \sum_{s=0}^{n-1}\left[\alpha u^{2}(s)+q g(s) u(s)\right]
$$

where $\alpha, p, q$ are nonnegative constants and $u$ and $g$ are nonnegative functions, and they estimated that

$$
u(n) \leq(1+\alpha)^{n}\left[p u(0)+\sum_{s=0}^{n-1} q g(s)\right]
$$

Another form of sum-difference inequality

$$
u^{p}(n) \leq c+\sum_{s=0}^{n-1}\left[f(s) g(u(s))+\sum_{\sigma=0}^{s-1} h(s, \sigma) g(u(\sigma))\right]
$$

where $c$ is nonnegative constant, and $u, f$ and $h$ are nonnegative functions, $g$ is nondecreasing function such that $g(u)>0$ on $(0, \infty)$, was estimated by Pachpatte [9] as

$u(n) \leq\left[G^{-1}\left[G(c)+\sum_{s=0}^{n-1}\left[f(s)+\sum_{\sigma=0}^{s-1} h(s, \sigma)\right]\right]\right]^{1 / p}$

where $G(r)=\int_{1}^{r} \frac{1}{g\left(s^{1 / p}\right)} d s$.

In [9], Pachpatte obtained following theorem:

Theorem 1.1 Let $u(m, n), f(m, n) \in D\left(N_{0}^{2}, R_{+}\right)$, $h(m, n, \sigma, \tau) \in D\left(E, R_{+}\right)$and $c \geq 0, p>0$ be real constants, where $E=\left\{(m, n, \sigma, \tau) \in N_{0}^{4}: 0 \leq \sigma \leq m<\infty, 0 \leq \tau \leq n<\infty\right\}$, if

$$
\begin{aligned}
& u^{p}(m, n) \leq c+\sum_{s=0}^{m-1} \sum_{t=0}^{n-1}[f(s, t) g(u(s, t)) \\
& \left.+\sum_{\sigma=0}^{s-1} \sum_{\tau=0}^{t-1} h(s, t, \sigma, \tau) g(u(\sigma, \tau))\right]
\end{aligned}
$$

for $m, n \in N_{0}$, then for

$$
\begin{aligned}
& 0 \leq m \leq m_{1}, 0 \leq n \leq n_{1} ; m, m_{1}, n, n_{1} \in N_{0}, \\
& u(m, n) \leq\left\{\Phi_{p}^{-1}\left[\Phi_{p}(c)+B(m, n)\right]\right\}^{\frac{1}{p}},
\end{aligned}
$$

Where

$$
\begin{aligned}
& B(m, n)=\sum_{s=0}^{m-1} \sum_{t=0}^{n-1}\left[f(s, t)+\sum_{\sigma=0}^{s-1} \sum_{\tau=0}^{t-1} h(s, t, \sigma, \tau)\right], \\
& \Phi_{p}(r)=\int_{r_{0}}^{r} \frac{1}{g\left(s^{1 / p}\right)} \mathrm{d} s, r>0,
\end{aligned}
$$

$r_{0}>0$ is arbitrary, $\Phi_{p}^{-1}$ is the inverse of $\Phi_{p}$ and $m_{1}, n_{1} \in N_{0}$ are chosen such that $\Phi_{p}(c)+B(m, n) \in \operatorname{Dom}\left(\Phi_{p}^{-1}\right)$, for all $m, n$ lying in $0 \leq m \leq m_{1}, 0 \leq n \leq n_{1}$.

In this paper, we offer some nonlinear discrete inequalities in two independent variables which can be used more conveniently in specific applications. Some applications 
are also given to study the behavior of solutions of certain partial difference equations.

\section{Main Results}

In what follow, we denote by $R$ the set of real numbers. Let $R_{+}=[0, \infty), N_{0}=\{0,1,2, \cdots\}$. We use the usual convention that the empty sum is taken to be 0 . Our main result is given in the following theorem.

Theorem 2.1 Let $u(m, n), a(m, n), \quad f(m, n) \in D\left(N_{0}^{2}, R_{+}\right)$, $h(m, n, \sigma, \tau) \in D\left(E, R_{+}\right)$and $c \geq 0, p>1$ be real constants, where $E=\left\{(m, n, \sigma, \tau) \in N_{0}^{4}: 0 \leq \sigma \leq m<\infty, 0 \leq \tau \leq n<\infty\right\}$, $g \in C\left(R_{+}, R_{+}\right)$be nondecreasing with $g(r)>0$ for $r>0$. If

$u^{p}(m, n) \leq c+\sum_{s=0}^{m-1} \sum_{t=0}^{n-1} a(s, t) u(s, t)+\sum_{s=0}^{m-1} \sum_{t=0}^{n-1}[f(s, t) g(u(s, t))$

$\left.+\sum_{\sigma=0}^{s-1} \sum_{\tau=0}^{t-1} h(s, t, \sigma, \tau) g(u(\sigma, \tau))\right] u(s, t)$

for $m, n \in N_{0}$, then for

$0 \leq m \leq m_{1}, 0 \leq n \leq n_{1} ; m, m_{1}, n, n_{1} \in N_{0}$,

$u(m, n)$

$\leq\left\{\Phi_{p-1}^{-1}\left[\Phi_{p-1}\left(c^{1-1 / p}+A(m, n)\right)+B(m, n)\right]\right\}^{\frac{1}{p-1}}$,

Where

$$
\begin{aligned}
& A(m, n)=\sum_{s=0}^{m-1} \sum_{t=0}^{n-1} a(s, t) \\
& B(m, n)=\sum_{s=0}^{m-1} \sum_{t=0}^{n-1}\left[f(s, t)+\sum_{\sigma=0}^{s-1} \sum_{\tau=0}^{t-1} h(s, t, \sigma, \tau)\right], \\
& \Phi_{p}(r)=\int_{r_{0}}^{r} \frac{1}{g\left(s^{1 / p}\right)} \mathrm{d} s, r>0,
\end{aligned}
$$

$r_{0}>0$ is arbitrary, $\Phi_{p}^{-1}$ is the inverse of $\Phi_{p}$ and $m_{1}, n_{1} \in N_{0}$ are chosen such that $\Phi_{p-1}\left(c^{1-1 / p}+A(m, n)\right)+B(m, n) \in \operatorname{Dom}\left(\Phi_{p-1}^{-1}\right)$, for all $m, n$ lying in $0 \leq m \leq m_{1}, 0 \leq n \leq n_{1}$.

Proof. Let $c \geq 0$ and define a function $z(m, n)$ by

$$
\begin{aligned}
& z(m, n)=c+\sum_{s=0}^{m-1} \sum_{t=0}^{n-1} a(s, t) u(s, t) \\
& +\sum_{s=0}^{m-1} \sum_{t=0}^{n-1}[f(s, t) g(u(s, t)) \\
& \left.+\sum_{\sigma=0}^{s-1} \sum_{\tau=0}^{t-1} h(s, t, \sigma, \tau) g(u(\sigma, \tau))\right] u(s, t),
\end{aligned}
$$

Then $z(0, n)=z(m, 0)=c, \quad u(m, n) \leq\{z(m, n)\}^{1 / p}, \quad z(m, n)$ is positive and nondecreasing for $m, n \in N_{0}$, and

$$
\begin{aligned}
& z(m+1, n)-z(m, n) \\
& =\sum_{t=0}^{n-1} a(m, t) u(m, t)+\sum_{t=0}^{n-1}[f(m, t) g(u(m, t)) \\
& \left.+\sum_{\sigma=0}^{m-1} \sum_{\tau=0}^{t-1} h(m, t, \sigma, \tau) \varphi(u(\sigma, \tau))\right] u(m, t) \\
& \leq \sum_{t=0}^{n-1} a(m, t)\{z(m, t)\}^{1 / p}+\sum_{t=0}^{n-1}\left[f(m, t) g\left(\{z(m, t)\}^{1 / p}\right)\right. \\
& +\sum_{\sigma=0}^{m-1} \sum_{\tau=0}^{t-1} h(m, t, \sigma, \tau) g\left(\{z(\sigma, \tau)\}^{1 / p}\right]\{z(m, t)\}^{1 / p} \\
& \leq\{z(m, n-1)\}^{1 / p}\left\{\sum_{t=0}^{n-1} a(m, t)+\sum_{t=0}^{n-1}\left[f(m, t) g\left(\{z(m, t)\}^{1 / p}\right)\right.\right. \\
& \left.\left.+\sum_{\sigma=0}^{m-1} \sum_{\tau=0}^{t-1} h(m, t, \sigma, \tau) g\left(\{z(\sigma, \tau)\}^{1 / p}\right)\right]\right\}
\end{aligned}
$$

Or

$$
\begin{aligned}
& \frac{z(m+1, n)-z(m, n)}{\{z(m, n-1)\}^{1 / p}} \\
& \leq \sum_{t=0}^{n-1} a(m, t)+\sum_{t=0}^{n-1}\left[f(m, t) g\left(\{z(m, t)\}^{1 / p}\right)\right. \\
& \left.+\sum_{\sigma=0}^{m-1} \sum_{\tau=0}^{t-1} h(m, t, \sigma, \tau) g\left(\{z(\sigma, \tau)\}^{1 / p}\right)\right]
\end{aligned}
$$

Keeping fixed $n$ in (2.8), setting $m=s$ and summing over $s$ from 0 to $m-1$, notice that

$$
\begin{aligned}
& \frac{z(m, n)-z(m-1, n)}{\{z(m-1, n-1)\}^{1 / p}}+\frac{z(m-1, n)-z(m-2, n)}{\{z(m-2, n-1)\}^{1 / p}}+\cdots \\
& \frac{z(1, n)-z(0, n)}{\{z(0, n-1)\}^{1 / p}}=\frac{z(m, n)}{\{z(m-1, n-1)\}^{1 / p}} \\
& +z(m-1, n)\left(\frac{1}{\{z(m-2, n-1)\}^{1 / p}}-\frac{1}{\{z(m-1, n-1)\}^{1 / p}}\right) \\
& +\cdots+z(1, n)\left(\frac{1}{\{z(0, n-1)\}^{1 / p}}-\frac{1}{\{z(1, n-1)\}^{1 / p}}\right)^{1 / p} \\
& -\frac{z(0, n)}{\{z(0, n-1)\}^{1 / p}} \geq \frac{z(m, n)}{\{z(m-1, n-1)\}^{1 / p}}-c^{1-1 / p} \\
& \geq\{z(m, n)\}^{1-1 / p}-c^{1-1 / p} .
\end{aligned}
$$

We obtain 


$$
\begin{aligned}
& \{z(m, n)\}^{1-1 / p} \leq c^{1-1 / p}+A(m, n) \\
& +\sum_{s=0}^{m-1} \sum_{t=0}^{n-1}\left[f(s, t) g\left(\{z(s, t)\}^{1 / p}\right)\right. \\
& \left.+\sum_{\sigma=0}^{s-1} \sum_{\tau=0}^{t-1} h(s, t, \sigma, \tau) g\left(\{z(\sigma, \tau)\}^{1 / p}\right)\right] .
\end{aligned}
$$

In particular, since $A(m, n)$ is nondecreasing in each variable, for any fixed $0 \leq \bar{m} \leq m_{1}, 0 \leq \bar{n} \leq n_{1}$,

$$
\begin{aligned}
& \{z(m, n)\}^{1-1 / p}=\left[\{z(m, n)\}^{1 / p}\right]^{p-1} \\
& \leq\left(c^{1-\frac{1}{p}}+A(\bar{m}, \bar{n})\right) \\
& +\sum_{s=0}^{m-1} \sum_{t=0}^{n-1}\left[f(s, t) g\left(\{z(s, t)\}^{1 / p}\right)\right. \\
& \left.+\sum_{\sigma=0}^{s-1} \sum_{\tau=0}^{t-1} h(s, t, \sigma, \tau) g\left(\{z(\sigma, \tau)\}^{1 / p}\right)\right]
\end{aligned}
$$

for all $m, n$ lying in $0 \leq m \leq \bar{m}, 0 \leq n \leq \bar{n}$.

Now applying Theorem A to the function $\{z(m, n)\}^{1 / p}$, we have

$$
u(m, n) \leq\{z(m, n)\}^{1 / p}
$$$$
\leq\left\{\Phi_{p-1}^{-1}\left[\Phi_{p-1}\left(c^{1-1 / p}+A(\bar{m}, \bar{n})\right)+B(m, n)\right]\right\}^{1 /(p-1)}
$$

for all $0 \leq m \leq \bar{m}, 0 \leq n \leq \bar{n}$. In particular, this gives

$$
u(\bar{m}, \bar{n}) \leq\left\{\Phi_{p-1}^{-1}\left[\Phi_{p-1}\left(c^{1-1 / p}+A(\bar{m}, \bar{n})\right)+B(\bar{m}, \bar{n})\right]\right\}^{1 /(p-1)} .
$$

Since $0 \leq \bar{m} \leq m_{1}, 0 \leq \bar{n} \leq n_{1}$ is arbitrary, this concludes the proof of the theorem.

Theorem 2.2 Let $u(m, n), a(m, n), f(m, n) \in D\left(N_{0}^{2}, R_{+}\right)$, $h(m, n, \sigma, \tau) \in D\left(E, R_{+}\right)$and $c \geq 0, p>q>0$ be real constants, where $E=\left\{(m, n, \sigma, \tau) \in N_{0}^{4}: 0 \leq \sigma \leq m<\infty, 0 \leq \tau \leq n<\infty\right\}$, $g \in C\left(R_{+}, R_{+}\right)$be nondecreasing with $g(r)>0$ for $r>0$; If

$$
\begin{aligned}
& u^{p}(m, n) \leq c+\sum_{s=0}^{m-1} \sum_{t=0}^{n-1} a(s, t) u^{q}(s, t)+\sum_{s=0}^{m-1} \sum_{t=0}^{n-1}[f(s, t) g(u(s, t)) \\
& \left.+\sum_{\sigma=0}^{s-1} \sum_{\tau=0}^{t-1} h(s, t, \sigma, \tau) g(u(\sigma, \tau))\right] u^{q}(s, t)
\end{aligned}
$$

for $m, n \in N_{0}$, then for

$$
\begin{aligned}
& 0 \leq m \leq m_{1}, 0 \leq n \leq n_{1} ; m, m_{1}, n, n_{1} \in N_{0}, \\
& u(m, n) \\
& \leq\left\{\Phi_{p-q}^{-1}\left[\Phi_{p-q}\left(c^{1-q / p}+A(m, n)\right)+B(m, n)\right]\right\}^{\frac{1}{p-q}},
\end{aligned}
$$

where $A(m, n), B(m, n)$ are defined as in Theorem 2.1, and $m_{1}, n_{1} \in N_{0}$ is chosen such that

$$
\Phi_{p-q}\left(c^{1-q / p}+A(m, n)\right)+B(m, n) \in \operatorname{Dom}\left(\Phi_{p-q}^{-1}\right)
$$

for all $m, n$ lying in $0 \leq m \leq m_{1}, 0 \leq n \leq n_{1}$.

Proof. For any $r>0$, define

$$
\psi(r)=g\left(r^{1 / q}\right) .
$$

Then clearly $\psi$ is nondecreasing with $\psi(r)>0$ for $r>0$; By (2.9)

$$
\begin{aligned}
u^{p}(m, n) \leq c & +\sum_{s=m_{0}}^{m-1} \sum_{t=n_{0}}^{n-1} a(s, t) u^{q}(s, t) \\
& +\sum_{s=0}^{m-1} \sum_{t=0}^{n-1}\left[f(s, t) \psi\left(u^{q}(s, t)\right)\right. \\
& \left.+\sum_{\sigma=0}^{s-1} \sum_{\tau=0}^{t-1} h(s, t, \sigma, \tau) \psi\left(u^{q}(\tau, \sigma)\right)\right] u^{q}(s, t),
\end{aligned}
$$

for any $m, n \in N_{0}$. Writing $v=u^{q}$, this becomes

$$
\begin{aligned}
v^{\frac{p}{q}}(m, n) \leq c & +\sum_{s=m_{0}}^{m-1} \sum_{t=n_{0}}^{n-1} a(s, t) v(s, t) \\
& +\sum_{s=0}^{m-1} \sum_{t=0}^{n-1}[f(s, t) \psi(v(s, t)) \\
& \left.+\sum_{\sigma=0}^{s-1} \sum_{\tau=0}^{t-1} h(s, t, \sigma, \tau) \psi(v(\tau, \sigma))\right] v(s, t),
\end{aligned}
$$

define

$$
H_{p}(r)=\int_{r_{0}}^{r} \frac{1}{\psi\left(s^{1 / p}\right)} \mathrm{d} s, r>0,
$$

Since $p / q>1$, it follows from Theorem 2.1 that $v(m, n)$

$$
\leq\left\{H_{p / q^{-1}}^{-1}\left[H_{p / q^{-1}}\left(c^{1-q / p}+A(m, n)\right)+B(m, n)\right]\right\}^{1 /\left(p / q^{-1)}\right.}
$$


$=\left\{H_{(p-q) / q}^{-1}\left[H_{(p-q) / q}\left(c^{1-q / p}+A(m, n)\right)+B(m, n)\right]\right\}^{\frac{q}{p-q}}$

for all $0 \leq m \leq m_{1}, 0 \leq n \leq n_{1}$. Now it is elementary to check by the definition $\psi, H_{p}$ in (2.11),(2.12) that

$$
\begin{aligned}
& H_{\frac{p-q}{q}}(r)=\Phi_{p-q}(r), \text { thus we have } \\
& v(m, n) \leq\left\{\Phi_{p-q}^{-1}\left[\Phi_{p-q}\left(c^{1-q / p}+A(m, n)\right)+B(m, n)\right]\right\}^{\frac{q}{p-q}} \\
& 0 \leq m \leq m_{1}, 0 \leq n \leq n_{1} .
\end{aligned}
$$

Or

$$
\begin{aligned}
& u(m, n)=v^{\frac{1}{q}}(m, n) \\
& \leq\left\{\Phi_{p-q}^{-1}\left[\Phi_{p-q}\left(c^{1-q / p}+A(m, n)\right)+B(m, n)\right]\right\}^{1 /(p-q)}
\end{aligned}
$$

for all $0 \leq m \leq m_{1}, 0 \leq n \leq n_{1}$. where $m_{1}, n_{1} \in N_{0}$ is chosen such that

$$
\Phi_{p-q}\left(c^{1-q / p}+A(m, n)\right)+B(m, n) \in \operatorname{Dom}\left(\Phi_{p-q}^{-1}\right)
$$

for all $0 \leq m \leq m_{1}, 0 \leq n \leq n_{1}$. An important special case of Theorem 2.2 is the following

Corollary 2.3 Let $u(m, n), a(m, n), f(m, n) \in D\left(N_{0}^{2}, R_{+}\right)$, $h(m, n, \sigma, \tau) \in D\left(E, R_{+}\right)$and $c \geq 0, p>1$ be real constants, where

$E=\left\{(m, n, \sigma, \tau) \in N_{0}^{4}: 0 \leq \sigma \leq m<\infty, 0 \leq \tau \leq n<\infty\right\}$, $g \in C\left(R_{+}, R_{+}\right)$be nondecreasing with $g(r)>0$ for $r>0$. If

$$
\begin{aligned}
& u^{p}(m, n) \leq c \\
& +\sum_{s=0}^{m-1} \sum_{t=0}^{n-1} a(s, t) u^{p-1}(s, t)+\sum_{s=0}^{m-1} \sum_{t=0}^{n-1}[f(s, t) g(u(s, t)) \\
& \left.+\sum_{\sigma=0}^{s-1} \sum_{\tau=0}^{t-1} h(s, t, \sigma, \tau) g(u(\sigma, \tau))\right] u^{p-1}(s, t)
\end{aligned}
$$

for $m, n \in N_{0}$, then for $0 \leq m \leq m_{1}, 0 \leq n \leq n_{1} ; m, m_{1}, n, n_{1} \in N_{0}$,

$$
u(m, n) \leq \Phi_{1}^{-1}\left[\Phi_{1}\left(c^{1 / p}+A(m, n)\right)+B(m, n)\right]
$$

where $A(m, n), B(m, n)$ are defined as in Theorem 2.2, and $m_{1}, n_{1} \in N_{0}$ is chosen such that

$\Phi_{1}\left(c^{1 / p}+A(m, n)\right)+B(m, n) \in \operatorname{Dom}\left(\Phi_{1}^{-1}\right)$

for all $m, n$ lying in $0 \leq m \leq m_{1}, 0 \leq n \leq n_{1}$. In particular, we have the following useful consequence.
Corollary 2.4 Let $u(m, n), a(m, n), f(m, n) \in D\left(N_{0}^{2}, R_{+}\right)$, $h(m, n, \sigma, \tau) \in D\left(E, R_{+}\right)$and $c \geq 0, p>1$ be real constants, where $E=\left\{(m, n, \sigma, \tau) \in N_{0}^{4}: 0 \leq \sigma \leq m<\infty, 0 \leq \tau \leq n<\infty\right\}$,

$$
\begin{aligned}
& u^{p}(m, n) \leq c+\sum_{s=0}^{m-1} \sum_{t=0}^{n-1} a(s, t) u^{p-1}(s, t)+\sum_{s=0}^{m-1} \sum_{t=0}^{n-1}[f(s, t) u(s, t) \\
& \left.+\sum_{\sigma=0}^{s-1} \sum_{\tau=0}^{t-1} h(s, t, \sigma, \tau) u(\sigma, \tau)\right] u^{p-1}(s, t),
\end{aligned}
$$

for $m, n \in N_{0}$, then

$$
u(m, n) \leq\left(c^{1 / p}+A(m, n)\right) \exp (B(m, n)),
$$

for all $m, n \in N_{0}$. where $A(m, n), B(m, n)$ are defined as in Theorem 2.2,

\section{III. an Application to boundary value problem}

The We consider the following Boundary value problem (BVP):

$$
\begin{aligned}
& \Delta_{12} z^{p}(m, n)=z^{p-1}(m, n) a(m, n) \\
& +z^{p-1}(m, n) M\left(m, n, z(m, n), \sum_{\sigma=0}^{m-1} \sum_{\tau=0}^{n-1} G(m, n, \sigma, \tau, z(\sigma, \tau))\right)
\end{aligned}
$$

Satisfying

$$
\begin{aligned}
z(m, 0) & =a_{1}(m), \\
z(0, n) & =a_{2}(n), a_{1}(0)=a_{2}(0)=0 .
\end{aligned}
$$

Where $p>1$ are given. The following theorem gives the bounded on solution of BVP.

Theorem 3.1. Consider (BVP). If

$$
\begin{aligned}
& |M(p, q, j, k)| \leq f(p, q) g(|j|)+|k| \\
& |G(m, n, r, s, t)| \leq h(m, n, r, s) g(|t|) \\
& \left|a_{1}(m)\right|^{p}+\left|a_{2}(n)\right|^{p} \leq c \quad \text { for some } c \geq 0,
\end{aligned}
$$

then all solutions of (BVP) satisfy

$$
|z(m, n)| \leq \Phi_{1}^{-1}\left[\Phi_{1}\left(c^{1 / p}+A(m, n)\right)+B(m, n)\right]
$$

Proof. It is easy to see that, the solution $z(m, n)$ of (BVP) satisfies the equivalent sun-difference equation

$$
\begin{aligned}
& z^{p}(m, n)=a_{1}^{p}(m)+a_{2}^{p}(n)+\sum_{s=0}^{m-1} \sum_{t=0}^{n-1} z^{p-1}(s, t) a(s, t) \\
& +\sum_{s=0}^{m-1} \sum_{t=0}^{n-1} z^{p-1}(s, t) M\left(s, t, z(s, t), \sum_{\sigma=0}^{s-1} \sum_{\tau=0}^{t-1} G(s, t, \sigma, \tau, z(\sigma, \tau))\right)
\end{aligned}
$$

From (3.7), (3.2), (3.3), (3.4), (3.5), we have 


$$
\begin{aligned}
& |z(m, n)|^{p} \leq c+\sum_{s=0}^{m-1} \sum_{t=0}^{n-1} a(s, t)|z(s, t)|^{p-1} \\
& +\sum_{s=m_{0}}^{m-1} \sum_{t=n_{0}}^{n-1}[f(s, t) g(|z(s, t)|) \\
& \left.+\sum_{\sigma=0}^{s-1} \sum_{\tau=0}^{t-1} h(s, t, \sigma, \tau) g(|z(s, t)|)\right]|z(s, t)|^{p-1} .
\end{aligned}
$$

Now a suitable application of the inequality (2.14) given in Corollary 2.3 to (3.8) yields (3.6).

\section{References}

[1] R. P. Agarwal, S. Deng, and W. Zhang, "Generalization of a retarded Gronwall-like inequality and its applications," Applied Mathematics and Computation, 165, 3, (2005).

[2] P. Y. H. Pang and R. P. Agarwal, "On an integral inequality and its discrete analogues," Journal of Mathematical Analysis and Applications, 194, 2, (1995).

[3] D. Bainov and P. Simeonov, Integral Inequalities and Applications, vol. 57 of Mathematics and Its Aplications, Kluwer Academic Publishers, Dordrecht, The Netherlands, (1992).

[4] S. S. Dragomir and Y. H. Kim, "Some integral inequalities for functions of two variables," Electronic Journal of Differential Equations, 10, (2003).
[5] O. Lipovan, "A retarded Gronwall-like inequality and its applications," Journal of Mathematical Analysis and Applications, vol. 252, 2, (2000).

[6] B. G. Pachpatte, "On some fundamental integral inequalities and their discrete analogues," Journal of Inequalities in Pure and Applied mathematics, 2, 2, (2001).

[7] B. G. Pachpatte, "On a certain retarded integral inequality and applications," Journal of Inequalities in Pure and Applied mathematics, $5,1,(2004)$.

[8] B. G. Pachpatte, "On some new nonlinear retarded integral inequalities," Journal of Inequalities in Pure and Applied mathematics, 5, 3, (2004).

[9] B. G. Pachpatte, "Integral inequalities of the Bihari type," Mathmatical Inequalities \& Applications, 5, 4, (2002).

[10] W. S. Cheung, J. L. Ren, "Discrete non-linear inequalities and applications to boundary value problems," Journal of Mathematical Analysis and Applications, 319, 1,(2006).

[11] W. S. Cheung, "Some discrete nonlinear inequalities and applications to boundary value problems for difference equations," Journal of Difference Equations and Applications, 10, 2, (2004).

[12] W. S. Cheung, "Some new nonlinear inequalities and applications to boundary value problems," Nonlinear Analysis: Theoy Methods \& Aplications, 64, 9, (2006).

[13] Sh. Salem and K. R. Ralan, "Some new discrete inequalities and their applications," Journal of Inequalities in Pure and Applied mathematics, $5,1,(2004)$. 